\title{
EL DESCIFRAMIENTO DE LA ESCRITURA DE LOS INKAS
}

Victoria de la Jara

\author{
Investigadora asociada al Museo de Arqueología y Etnología \\ de la Universidad Nacional Mayor de San Marcos
}

Cuando el hombre contemporáneo perdió la emoción y expectativa por el descubrimiento de mundos nuevos y exóticos, la Arqueología, con sus hallazgos sorprendentes de las últimas décadas, canalizó parte del interés humano por lo desconocido, que puede darle una enseñanza. El antiguo Perú es la promesa del futuro. La vegetación devuelve ciudades inmensas y en las cumbres de las más altas montañas hay templos a los que el hombre contemporáneo asciende lentamente. La verdad emerge también de una historia falseada para disimular delitos o negligencias, pero los científicos e historiadores vamos exhibiendo al mundo la verdadera imagen del Tahuantinsuyo, el imperio del hijo del Sol.

\section{El DESCUBRIMIENTO DE LA ESCRITURA DE LOS INKAS}

El imperio de los Inkas sigue ejerciendo extraña fascinación sobre los historiadores de todo el mundo. El Inka fue un príncipe aislado, que en un continente lejano realizó un programa de justicia agraria y seguridad social, sin la presión de las dolorosas crisis sociales contemporáneas $\mathrm{o}$ los hechos aleccionadores que siguieron a la caída de la VI Dinastía egipcia. Los peruanos llegan a la justicia social antes que los euro- peos, que destruyeron el sistema porque aún no podrían comprenderlo

El prestigio del príncipe peruano exhibía ante la historia su único defecto: no había "alcanzado a escribir". Esta idea se repetía en todos los libros y llegó a convertirse en un mito tan poderoso, que los riesgos y peligros de destruirlo hacían vacilar a los más valientes. Los sabios desconocían que no había ningún estudio sobre el problema de la existencia de la escritura de los inkas, y que el escepticismo sólo tenía el respaldo de la lectura de viejos libros de los siglos XVI y XVII, o sea escritos siglos antes de los primeros estudios científicos sobre las escrituras primitivas.

El descubrimiento de las escrituras perdidas suele coincidir con un hallazgo arqueológico, pero el sistema gráfico inka permanecía desconocido pese a que sus textos se exhibían en museos. Ello dio motivo a que algunos científicos sintieran indignación ante mis trabajos y en los primeros años fui víctima de un verdadero sabotaje.

En una década reuní una amplia documentación de textos inkas en tejidos, keros y también en cuadros de la época colonial, que reproducen personajes peruanos con vestidos con inscripciones. Estudiando las palabras 
de los viejos vocabularios de la lengua de los inkas, y comparándolas con las descripciones de los antiguos relatos, descubrí que coincidían al referirse a una escritura multicolor, con signos en forma cuadrada y a textos obre tejidos, madera y otros materiales. Identifiqué los signos cuadrados en el material arqueológico y mis catálogos y estudios estructurales probaron la existencia de la escritura aún en épocas de la dominación española.

Corresponde al científico francés M. Marcel Cohen el mérito de haber sido el primero en haber aceptado la existencia de la escritura de los Inkas, después de examinar un amplio informe con los resultados de mi trabajo, y que -dedicado a su nombre- la remití en el año 1966. Mi primer artículo fue también publicado en Francia (Science-Progress, Juillet 1967).

\section{El Primer Congreso del Hombre y la Cultura Andina}

El los primeros días del presente año se realizó en Lima un importante Congreso organizado por el Museo de Arqueología y Etnología de la Universidad Nacional Mayor de San Marcos, y en él muchos científicos peruanos tuvimos la oportunidad de presentar los resultados de nuestras investigaciones, que permanecían inéditas y casi desconocidas por la escasez de publicaciones en el Perú. Quedó demostrado que son muchas y muy importantes las aportaciones de los científicos peruanos al conocimiento de nuestras antiguas civilizaciones.

En el XXXIX Congreso Internacional de Americanistas, realizado en Lima en el año 1970, presenté un trabajo sobre la escritura en el antiguo Perú, con una fundamentación tan amplia, que mereció posteriormente el Premio Nacional de Fomento a la Cultura, pero -por motivos difíciles de comprender- el Congreso de Americanistas motivó la creación de un nuevo mito, según el cual mi investigación quedaría reducida a las etapas iniciales. Pocos comprendieron, aunque era lógico suponerlo, que si había descubierto la escritura de los inkas y sabía valorara la importancia de este hecho, aspiraría a culminar mi obra con el desciframiento. Para el éxito de esta segunda eta- pa unía mi experiencia de 10 años examinando los signos inkas y los estudios realizados en le Universidad de México, que gentilmente me invitó en el año 1968, para conocer los métodos de desciframiento en la escritura maya.

En el último año dupliqué mi documentación de keros con inscripciones y analizándolos sistemáticamente, con el aporte de datos míos muy antiguos, logré amplios resultados en la lectura de los signos inka. El profundo secreto que rodeó mi trabajo, del cual sólo conocieron tres amigos peruanos, motivó mi ponencia en el Primer Congreso Peruano del Hombre y la Cultura Andina, polarizara la atención de los científicos y periodistas que asistían a las sesiones. Como el mejor aporte de mi trabajo pude leer un epitafio u oración fúnebre al célebre inka Pachacuti, y escrito en bellos signos multicolores.

\section{EL ENIGMA DE LOS KEROS INKA}

Los keros son vasos de madera usados por los inkas en el antiguo Perú y sus descendientes. Sólo se fabricaban en la región del Cusco, capital del imperio. Las figuras coloreadas de los keros están incrustadas en las maderas previo diseño grabado y cubierto con una goma o mastic.

Sólo un 10\% de los keros tienen inscripciones y ellas se asocian siempre a escenas de gran valor histórico, ceremonial o evocativo. La fabricación del pan o el entierro de un campesino están representados en los keros, pero no acompañados de inscripciones (Fig. 0).

Aún en keros de fabricación colonial, comprobé la ausencia de la cruz cristiana. Este hecho en 2000 keros señala un rechazo intencional, porque representaron el caballo, la sirena y la guitarra que también eran foráneos.

Los keros no fueron ofrenda fúnebre. Los heredaron las familias imperiales que los conservaban como un bien sagrado, que se negaban a vender. Los keros se fabricaban por pares y hasta ahora existe la costumbre de ofrecer un vaso igual para una libación con un huésped. Tenemos el relato de destrucción de keros 
por los extirpadores de idolatrías y este hecho señalaba que su valor religioso o evocativo se consideró como peligroso.

Observé que algunos signos acusaban una frecuencia mucho más elevada según la posición de los signos y las escenas en la superficie del kero y estructuré mi tipología del kero-texto -la primera que existe- para comprobar la relación entre el signo y el tipo de kero.

El análisis estructural de las inscripciones y el estudio de la iconografía de los keros tipo B-5-(a) me dio datos exactos sobre la relación del texto-escena -que no había sido descubierta- y me abrió la brecha para una amplia lectura de inscripciones en keros.

En el reciente congreso del Museo de San Marcos pude resumir mis trabajos sobre desciframiento de keros con estas palabras: "No hay dos keros con inscripción igual, salvo que sean pareja, o dos signos sin importancia. Cada kero evoca un personaje, un palacio o templo y un pedazo de historia que ni pudo ni quiso ser olvidada. En los keros está una historia escrita por los inkas. La lectura de las inscripciones en keros restituirá fragmentos de los famosos códices de madera que algunos creen perdidos, y que según los viejos relatos fueron mandados hacer por el inka Pachacuti para memoria de los forjadores de la grandeza del imperio".

No era lógico suponer que un pueblo que tenía historiadores descuidara el legar a sus descendientes su propia versión dejando al extranjero o mestizo la tarea de relatar los hechos más importantes de su pasado. La fabricación tardía de algunos keros me lleva a pensar que los códices de Pachacuti fueron salvados y examinados por los descendientes de los inkas hasta fecha no muy lejana.

\section{LA SIMBOLIZACIÓN DEL SEXO EN LOS KEROS}

En los keros tipo B-5-(a) la escena -aparentemente igual- está asociada a inscripciones visiblemente diferentes, aunque en algunos casos se observa una misma secuencia de tres signos. Pude descubrir las leyes que rigen este cambio de inscripción o su repetición. También explican la presencia o ausencia de los signos:
1. Varía la inscripción si la flor que una mujer ofrece al inka es de color rojo o blanco;

2. Un signo está presente o ausente en la inscripción coincidiendo con la presencia o ausencia de un "picaflor" junto a la flor ofrecida al Ica;

3. Hay cambios en los signos de la inscripción, si el inka está de pie o sentado, ante la mujer que le ofrece la flor. Otros cambios en la escena rigen también la modificación del texto. El análisis de lo sueros tipos B-5(a) me dio el testimonio del simbolismo de las escenas -aparentemente iguales-y del valor de los colores.

La presencia o a usencia del "picaflor" y su relación con un signo de la escritura de los inkas, me llevó al descubrimiento de la representación simbólica del sexo en los keros. Sabemos que desde las cuevas prehispánicas el triángulo invertido es símbolo femenino y la pluma es el sexo masculino. Analizando los motivos florares de los keros pude identificar en ellos los símbolos sexuales, que son -en pueblos que fincaron su esperanza de prosperidad económica en la agricultura- un culto a la fertilidad. El arte egipcio no representó escenas eróticas -en milenios de cultura- pero exhibe el culto del dios Min. (Fig 1)

El descubrimiento de la simbolización del sexo en los keros es muy importante, porque existe en América una escritura de formas pictográficas, que fue muy usada por los sacerdotes jesuitas en su tarea de evangelización, siendo oraciones católicas casi todos los textos descifrados. La fabricación en época colonial de algunos keros señalaba la posibilidad de que existiera algún rito semicristiano y este hecho era importante para el desciframiento de las inscripciones en keros. La ausencia de la cruz cristiana -en todos los keros que examiné- y la simbolización del sexo en vasos rituales señalaron la persistencia clandestina del culto a los antiguos dioses, y sus nombres debían ser buscados en los textos.

\section{El IMPERIo InKA y el SIMbolismo de TAHUA}

Para descifrar una escritura fuera de uso buscamos en los textos los nombres de los dioses, 
dignatarios y ciudades importantes. Los métodos modernos perfeccionaron los sistemas de lectura, que son conocidos desde el siglo XIX. La intuición del científico suele guiarlo en la elección acertada de las "palabras probables". Si ella son erradas el desciframiento no prospera. Con las palabras descifradas debemos leer palabras compuestas, inscripciones y textos.

El estudio de la historia de los inkas revela que el número cuatro o tahua, tuvo un gran valor simbólico, dando origen a varios mitos y al nombre del imperio o Tawantinsuyo: reino de las cuatro regiones del mundo.

$\mathrm{Al}$ iniciar mi desciframiento de las inscripciones en keros separé los signos cuyo dibujo representaba la cifra 4 y que estaban ordenados en mi catálogo, obteniendo el siguiente resultado: (Fig. 2).

1. CUSCO: Signo de muy alta frecuencia. Siempre de color rojo en todos los keros. Cuatro pequeños cuadritos de color variable señalaban los cuatro puntos cardinales, característica de la ciudad del Cusco.

2. PACHA (mundo, tiempo): signo de frecuencia limitada. Siempre de color rojo en todos los keros. Cuatro pequeños semicírculos de color variable señalan los cuatro ángulos del cielo y la tierra.

3. SAPAN INKA (único rey): signo de muy alta frecuencia. Siempre de color rojo en todos los keros. Cuatro plumas de ave sagrada (usadas por el inka) señalan los 4 ángulos del mundo. Si las plumas no son negras es príncipe o noble pero el soberano.

4. INTI (dios Sol): signo de frecuencia limitada. Totalmente rojo o en un $50 \%$ por motivos que explicaré. Cuatro rayos cruzan el signo Pacha.

5. CAMAC O CAPAC (creador o poderoso): signo escalonado. Muy alta frecuencia. Muchas variantes de color y pequeñas modificaciones en el dibujo. Es el signo que se asocia al "picaflor" en los keros tipo B-5(a).

6. WIRACOCHA (dios relacionado a la fertilidad): signo de frecuencia limitada. Está formado con la unión de cuatro signos $\mathrm{Ca}$ mac o Capac dejando al centro un pequeño dibujo que evoca al huevo con el cual el dios estuvo representado en el templo del Cusco. Variante de este signo parecen formar el nombre del imperio o Tawantinsuyo.

Los signos Sapan Inka y Cusco son una frecuencia muy elevada en las inscripciones en keros porque éstas se refieren a hechos o personajes históricos. Por este motivo son también signos de alta frecuencia los de las palabras apu (gran señor) y hatun (grande).

\section{LOS SIGNOS DEL CIELO DE LOS INKAS O JANAN PACHA}

Ya en mi kero № 12 -de los más antiguos de mi serie- descifré los signos del dios o Inti y la diosa Luna, o Mama Quilla, pero el trabajo quedó abandonado y volví a él con una nueva investigación. En la actualidad todos los signos del Janan Pacha (mundo de arriba) están descubiertos por mi retorno a la lectura del signo Inti, que es el "aspa" descrita por un antiguo cronista, que señala su presencia entre los ídolos de los inkas.

Examinando la lámina de colores que acompaña el presente trabajo observamos porqué el signo Pacha (mundo, tiempo) me llevó al desciframiento de los nombre de los dioses que tuvieron sus altares en el Templo del Sol del Cusco. Ellos son: Inti (el dios Sol), Chasca (Venus), Onkoy (constelación), illapa (rayo), Koychi (arco iris). El signo cocha (lago) pertenece al mismo grupo y estaba representado en el altar del Cusco. Muchos pueblos creyeron que las lluvias se debían a la caída del agua de un gran río o lago celestial. La Luna o Mama Quilla pertenece al grupo de los signos representados por los triángulos femeninos.

Con el análisis de los keros tipo B-5-(a) puede comprobar que el color de la flor ofrecida al inka rige el cambio de la inscripción del kero. El rojo sagrado de los inkas es también inalterable en los signos Cusco, Pacha e Inka. Estos datos me llevaron a analizar los cambios de color del signo Inti (dios Sol) y descubrí que con los cambios de color se obtiene un amplio repertorio de palabras. 
El dios Sol de los inkas tenía tres nombres, de acuerdo a los viejos relatos: era Anti al levantarse en la madrugada, y en el signo lo señalan los colores de la luz y la sombra: era Inti en el cenit y lo representa un signo rojo, y era Konti en el ocaso y de color rojo, el sol se sumerge en las sombras. Los signos representan claramente estas variantes mediante sus colores.

El signo Inti señala también los solsticios y los equinoccios. El color rojo en el centro del signo señala que en este punto el horizonte se levanta y se pone el Sol en los equinoccios. La primavera y el otoño son equinoccios en el Cusco y se les identifica por los colores del signo. El rojo horizontal del Inti señala los solsticios, ya que en ellos se levanta y se pone el Sol en un extremo del horizonte. Por los colores se conoce también el signo del verano y el del invierno. El Inti de verano es muy visible en el interior del signo del Arco Iris, y es en esta estación que se le ve en el cielo del Cusco. El color azul en el signo señala las épocas de lluvia y riego y los colores castaños las épocas secas. Con estos signos creo posible identificar las fiestas agrícolas representadas en los keros y que se celebran en determinados meses del año. Pueden darnos datos sobre la astronomía incaica en la cual los viejos relatos sólo señalan ideas generales.

El signo Chasca (Venus) es igual al signo Maya, pero aquél carece de color. Puede ser una simple coincidencia ya que el signo de la Luna es también muy semejante en los inkas y los sumerios. En la escritura inka las variantes de color en los ángulos del signo de Venus señalan su diferencia como estrella del amanecer o atardecer y se obtienen así las palabras Punchao (el día) y Tuta (la noche).

La Mama Quilla o Luna unida al Sol es un signo, representa el Eclipse. Los cambios de color en el signo de la Luna señalan toda la lunación. Con el signo Cocha (lago) se escriben las palabras Capac Cocha (gran sacrificio inka), y Hatun Cocha (gran lago).

La comprobación de lectura correcta con estos signos es muy amplia. Con el logograma Pacha se forman las palabras Pacha Mama (la tierra), Pacha Yachachic (dios maestro del mundo) y Hatun pacha (tiempo de cosecha, según los antiguos vocabularios). El signo Pachacuti, es el Pacha dividido en dos por una línea horizontal (Fig. 3).

El número de signos registrado en mis catálogos y las características de las inscripciones revelan que la escritura de los inkas en un sistema logográfico, en el cual cada signo representa una palabra, pero al usarse los colores para variar el significado del signo se redujo el número de formas básicas. En inka impuso el uso de su lengua (el runa simi o lenguaje del hombre). Era hablado en todo su vasto imperio, que hoy compartimos varias repúblicas. Un sistema con signos palabras facilitaba el aprendizaje entre los dignatarios de las regiones recién conquistadas. Es conocido que las leyes del Cusco eran aplicadas en forma exacta en todo el imperio. Los códigos debieron existir en todas las regiones, y ello de la esperanza de poder recuperar alguno pues los del Cusco fueron destruidos por los españoles.

Los colores de los signos del cielo inka o Jatun pacha (el mundo de arriba) expresan claramente la realidad: el azul es agua y el castaño tierra seca. El negro es sombra y el rojo, blanco y amarillo son colores de luz. Hay en el cielo tres luces: la del Sol, la Luna y las Estrellas. Los signos astronómicos inka no habían sido descubiertos, pese a ser iguales en forma al de Venus para mayas e inkas. No sólo estaba perdida la escritura sino también los calendarios.

\section{SigNOS PARA TÍTULOS Y JERARCAS}

Para leer las inscripciones en keros es necesario conocer los signos que señalan las jerarquías sociales y religiosas masculinas y femeninas. Un progreso muy grande en mi tarea de desciframiento fue el descubrir estos signos.

Ya al hablar de la simbolización del sexo en los keros señalé que los triángulos son representación femenina y las plumas señalan el seco masculino. Así el signo Sapan Inka (único inka) es rojo y con cuatro plumas negras del ave sagrada, Si las plumas son de otro color no es el emperador sino un príncipe, considerando que el título inka era usado por los hijos y parientes cercanos del Sapan Inka. Una plu- 
ma -también sobre rojo- es Sinchi, guerrero, valeroso. Duplicando el dibujo de la pluma se escribe la palabra Sinchicuna (plural de sinchi).

Triángulos femeninos permiten escribir las palabras Ocllo (señora muy respetable), Nusta (princesa), Mama (madre) y su plural Mamacuna.

El análisis de la lámina en color revela que variedades del signo $A p u$ (gran señor) señalan su diferencia con el Auqui (señor) y el Sumac (el más grande de todos los Apus). Los cambios de color modifican el signo, pero siempre en una misma idea general. Si el signo Apu carece de la banda central es signo femenino como Palla (gran señora), Aclla (mujer escogida) y Warmi (esposa). Una variación de estos signos femeninos señala la jerarquía religiosa (Color C).

La presencia simultánea de los signos Apus y Sapan Inka revelan que en el kero está escrito el nombre de un emperador. No hay en estas inscripciones signos con triángulos femeninos. Los signos Palla, Aclla y signos con triángulos revelan estar el signo de inka o del Inti precedido del signo Warmi (esposa) ya que señalan el parentesco de la mujer con un personaje. Los signos de la jerarquía religiosa nunca están asociados al Sapan Inka o al Apu, tampoco a los signos de las damas. Ellos están casi siempre en keros sin escenas y cerca del Sol, la Luna, el Rayo o el Eclipse. Si cerca de signo hay triángulos es sacerdotisa. Sin triángulos y con los lados del signo más complicados es sacerdote. La lectura correcta de estos keros será la más difícil porque posiblemente contienen fórmulas sagradas conocidas por el clero. (Figs. 4 y 5 ).

En la Fig. 4 leemos el nombre del emperador Tupac Inka Yupanqui. Según los antiguos relatos este rey viajó a la Polinesia y consultaba frecuentemente a los ídolos del Lago Titicaca. Los nombres de Mama Ocllo y Mama Huaco son de reinas famosas. Aclla Huasi es el nombre del convento de las Vírgenes del Sol. Aclla significa mujer escogida y Huasi casa. Aclla Pampa era la pampa o pequeña propiedad agrícola de las mujeres escogidas. Apu Inti, Apu Inka y Capac Apu, son títulos que leemos frecuentemente en las viejas crónicas.
Próximamente publicaré una amplia lista de nombres de reyes y princesas del antiguo Perú escritas con sus signos multicolores y que revelan el gran valor histórico de los keros. El cronista mestizo Huamán Poma de Ayala y el escriba de los keros usan una redacción tan similar que revelan copia de un original común. En mi opinión aún existen los tablones del Puquin Cancha. La llegada de los españoles al Cusco no fue inmediata y su vandalismo en el templo costeño de Pachacamac debió prevenir a los amautas de la conveniencia de salvar su patrimonio cultural, que para ellos no era el oro. Hace 50 años no se conocía Machu Picchu y la escritura de los inkas estuvo perdida hasta 1962, fecha de mis primeros trabajos.

\section{LOS SIGNOS DE CIUDADES Y EDIFICIOS}

Aún existe el Cusco Imperial. Calles estrechas con pavimento empedrado nos llavan a lugares santos. El cristiano va a Jerusalén, el mahometano a La Meca y los mestizos peruanos llegamos reverentes a los muros indestructibles de nuestra antigua capital.

Existe el Cusco de los inkas y podemos caminar a la sombra del muro altísimo del Aclla Huasi, la casa de las Vírgenes del Sol. Allí recibieron educación esmerada centenares de jóvenes peruanas. El Yachay Huasi o "casa de enseñanza" era el centro de educación para los varones, siendo calificado por los antiguos cronistas como una verdadera Universidad. Existen también muros de palacios reales o Inka Huasi. Ni los avisos luminosos que anuncian la venta de cámaras fotográficas, ni las rejas, arquerías y miradores musulmanes destruyen la belleza monumental del Cusco de los inkas. Los viejos libros conservaron los nombres de sus templos y palacios. Muchos de ellos fueron construidos en la época del emperador Pachacuti, el más célebre de los inkas.

Los signos de las construcciones arquitectónicas son muy similares al signo "casa" de los jeroglíficos egipcios. La lectura de los signos Llajta (pueblo, ciuada) y Huasi (casa me llevó al desciframiento de los nombres de los grandes palacios, templos y edificios cuzqueños. Yachay Huasi (Universidad para varones), Ac- 
Ila Huasi (monasterio de las escogidas) e Inka Huasi (palacio real) son palabras de lectura fácil. Huaca + Huasi es tumba o casa del muerto, considerando que la palabra huaca también significa llorar. Este signo pertenece a un grupo amplio y que se identifican por dibujos escalonados. Wiracocha + Huasi es el templo del dios de ese nombre, construido por el padre del inka Pachacuti. En la Fig. 6 observamos que el palacio real y los grandes templos coinciden en una variante del signo huasi. Yupanqui + Huasi es el templo del Sol, no sólo por las momias de los inkas veneradas allí, sino porque la palabra Yupanqui (memorable) viene de yupana, que significa contar, y los dioses eran los astros que les permitían calcular el tiempo. El signo Yupanqui pertenece al mismo grupo del Tupac (resplandeciente). Este signo es una modificación del Illapa (rayo) por la semejanza de su brillo con el del Sol y la Luna. Esta forma de escribir los nombres de los edificios es muy similar al jeroglífico hitita en que la palabra dios única a casa se lee Templo.

La interpretación de Capac + Huasi es más difícil por ser el primer signo uno de los que ofrece más variantes de color y pequeñas modificaciones del dibujo. El signo huasi no señala gran templo y considerando que las inscripciones en keros se refieren a personajes o edificios famosos creo que se debe leer como el Museo Histórico o Puquin Cancha en el cual estaban guardados los grandes tablones con la historia de los Inkas mandada "pintar" por el inka $\mathrm{Pa}$ chacuti. Podría ser también la casa de los camayos o jefes como Camayoc + Huasi.

En 220 keros sólo encontré 7 veces el signo huasi. Están en vasos muy diferentes, que revelan ser obra de varios artistas. Los signos tienen la parte central dividida en cuatro partes. Los colores son rojo y azul y ocupan el mismo lugar en los 7 keros. En el palacio real del Cusco vivían las autoridades de las cuatro regiones del imperio o Suyos. El signo del templo en tejido representa la división central y los colores. El signo Llajta (pueblo, ciudad) expresa la idea de amplitud por carecer de la división central (Fig. 6).

CUSCO HATUN LLAJTA (Cusco gran ciudad) es una frase más amplia que el Hatun Cusco (gran Cusco) que se lee frecuentemente en los keros. Ambas son frases que se repiten mucho en los viejos relatos. El signo Cusco permite estudiar el proceso se simplificación de la forma: en el período más reciente el signo es rojo y sólo señala los cuatro puntos cardinales. En madera -pero más antiguo- el signo señala también las cuatro direcciones de la Pacha o mundo. En tejido el signo es más complicado. Se percibe en su borde la división de Hanan Cusco y Urin Cusco (Cusco de arriba y Cusco de abajo). Se ven los cuatro caminos reales hacia los cuatro puntos cardinales y las direcciones del mundo. Los campos centrales resultan ocho, que es el número mágico del Cusco. Los keros tienen frecuentemente inscripciones de ocho signos (Fig. 6). Mi método de identificar en los tejidos los signos que ya descifré en keros será la ruta hacia un desciframiento más amplio de los textos inka. El número de signos de los keros permite clasificarlos y el estudio de la frecuencia de los signos y de sus secuencias relacionadas con las escenas permite una comprobación de lectura correcta amplia. El número de signos de los tejidos es mayor, pero no hay escenas que guíen para la interpretación y la variedad de temas tratados en ellos se revela por los signos diferentes, siendo escasos los que se ven en casi todos los textos. En un caso contrario al de los keros con inscripciones cortas pero muchas de ellas semejantes.

\section{El ePITAFio u ORACión fúnebre De Pachacuti}

El más célebre de nuestros inkas es también uno de los más grandes legisladores del mundo. Solón o Licurgo, Hamurabi o Ur-Nanmu no llegaron a comprender como Pachacuti que el robo de un hombre hambriento era delito de las autoridades, cuya negligencia debía ser castigada.

Pachacuti significa ciclo de 500 años. Pacha es mundo, pero también tiempo. El cronista mestizo Garcilaso de la Vega escribe que el nombre del inka Pachacuti significa "el que vuelve" o "el que cambia el mundo" ¿Creyeron los sabios sacerdotes del imperio de un retorno de los acontecimientos, como lo esperaban los mayas?, iconfiaron en que un ciclo de 500 años o sea un Pachacuti sería suficiente para superar los estragos sufridos por la llegada de Pizarro? 
Historiadores clandestinos copiaron en los keros de fabricación colonial escenas históricas con sus batallas de reyes y princesas. Lo cristiano es rechazado. Hay en los keros sirenas, pero no vírgenes católicas. Ningún kero evoca la construcción de la catedral del Cusco, pero sí la guerra de los inkas Huascar y Atahualpa. Hay muchos keros con fiestas al dios Sol, pero jamás la cruz cristiana. Mis desciframientos revelan que los nombres de inkas y princesas fueron escritos con sus bellos signos multicolores como si hubiera existido la convicción de que podrían ser comprendidos en el futuro. No revelaron el secreto del sistema, pero escribieron.

De los 220 keros de mi serie, el № 24 es el más interesante. De indiscutible autenticidad comparte sus características sólo con el kero $n^{0}$ 54. Son del tipo A, o sea sin escenas. Con tres hileras de signos en la tercera hilera, siendo diferentes los signos de la hilera central. La inscripciones de los dos keros son también diferentes y uno tiene 30 signos y el otro 33.

Hay dos lecturas posibles para el kero $\mathrm{n}^{\mathrm{o}}$ 24. La totalidad del texto de 30 signos, que evocan la semana inka de 10 días, que multiplicados por 3 formaban el mes, y la lectura de los doce signos de la parte central que evocan los 12 meses de año. Esta segunda lectura se establece por la simetría que forman los signos en su cambio de color. El primer signo de la Luna y el primer signo del Inti son también de otro color. En la primera hilera del kero $\mathrm{n}^{\mathrm{o}}$ 24 se lee: madre fértil Apu Inti resplandeciente luna creador Apu Inti radiante. Los cambios de color en los adjetivos señalan sinónimos. En la tercera hilera están escritas estas palabras: Inti luminosos madrecita fértil Sumac Inti resplandeciente luna creador Apu. Con signos ya muy conocidos y rodeada de esta oración mística se lee esta inscripción en la hilera central: tumba del gran Pachacuti cusqueño Sinchi Pachacuti gran cusqueño Sinchi.

La lectura central de 12 signos tiene en la hilera primera y tercera esta oración abreviada: Apu Inti resplandeciente luna creador Apu Sumac Inti. Estas palabras parecen envolver como una protección sagrada una inscripción de lectura más breve: Pachacuti cusqueño Sinchi Pachacuti.
La palabra sinchi es de traducción difícil. Significa valeroso o señala rango militar, pero delante de un nombre indica su gran importancia. Creo posible descubrir en esta inscripción una fórmula matemática de 500 o sea un ciclo Pachacuti. Un cronista antiguo relata que en el templo del Sol del Cusco había una tela con la historia de Pachacuti y que en ella le leyeron sus grandes obras y los años de su vida y reinado. La inscripción fúnebre del kero no 24 es copia de algún documento antiguo destruido por los españoles y salvado parcialmente al ser copiado por consejo de un sabio amauta.

Los cambios de color de los signos permiten conocer el comienzo de la inscripción. Hay tres signos de la Luna diferentes, y sólo uno de ellos se duplica. Del signo Inti o Sol hay tres iguales y uno diferente y lo mismo se observa en el signo Apu. Cuando el adjetivo que señala la fertilidad es aplicado a la Luna tiene el mismo color, que cambia al aplicarse al Apu, que por ser signo masculino no es fértil, sino creador. El signo que significa resplandeciente es igual para la Luna, pero es la palabra radiante o luminoso para el Sol o Inti.

El color de la flor ofrecida al inka en los keros tiene B-5-(a) rige el cambio de la inscripción. El color de los signos Inti y los astros señalan los cambios de estación y horas del día. Las variantes de color de los signos que son adjetivos señalan cambios gramaticales. El runa simi o lengua del inka no tiene artículos. Es aglutinativo o yuxtapositivo. Emplea sufijos y en una sola palabra pueden estar representadas diferentes partes de la oración. El signo de escritura inka exhibe variantes de color con leyes fijas. Pequeñas variantes de dibujo con adición de líneas, bandas y puntos. Signos de color inalterable o variable. Gramática de colores, tan sorprendente como el imperio mismo, como su arquitectura incré́ble, como sus leyes sabias, como su justicia agraria que no fue ejemplo porque la destruyeron. Como todo en el Perú fascinante, pero perdido u olvidado, como un amauta sabio que debió soñar con el siglo XXI, porque en él se cumple el ciclo. Con él comenzaremos un nuevo Pachacuti y como una promesa el epitafio del inka se descifra cuando está próximo a terminar el siglo XX. Pachacitu: "el que vuelve". 


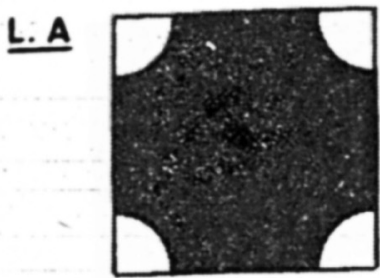

EL MUNDO TIEMPO

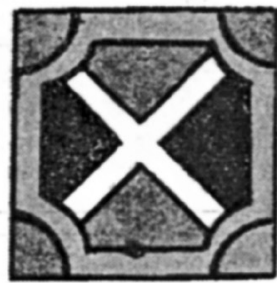

ARCO IRIS

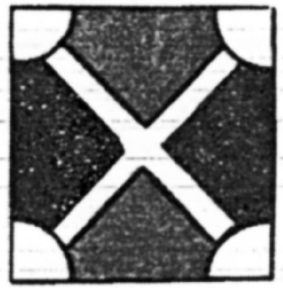

VERANO

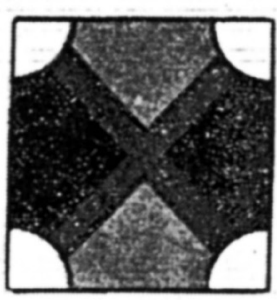

SOL LEVANTE

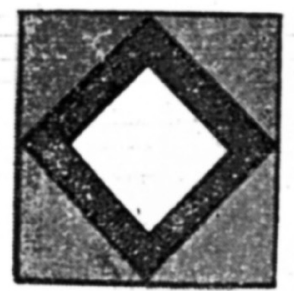

LUNA LLENA

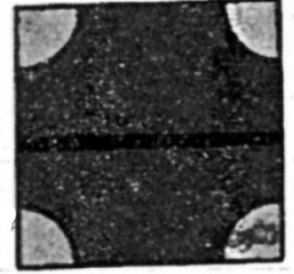

500. ANNOS

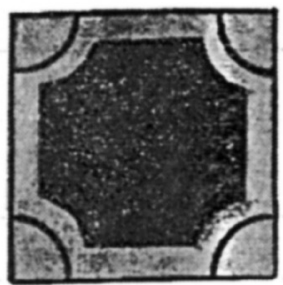

EL LAGO

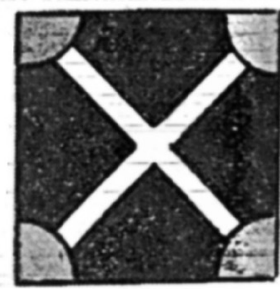

INVIERNO

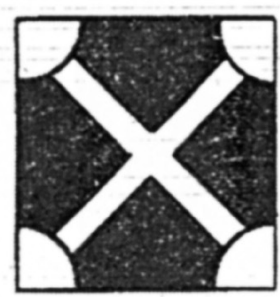

CENIT

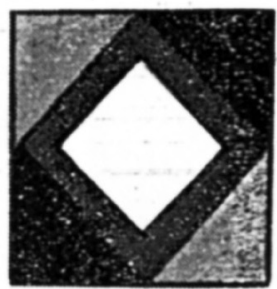

LUNA MENGUANTE

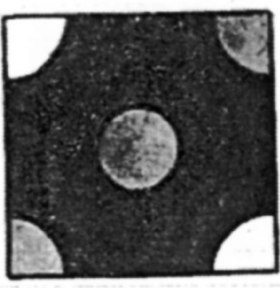

EL DIA

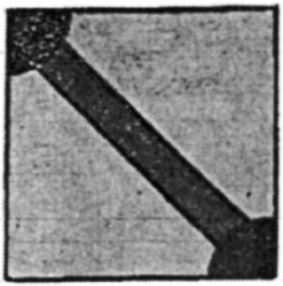

EL RAYO

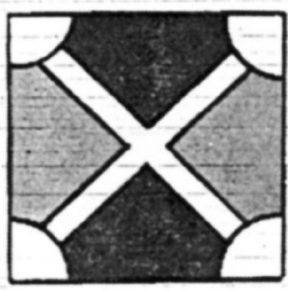

PRIMAVERA

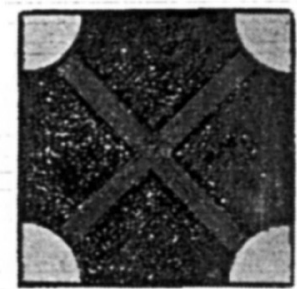

SOL PONIENTE

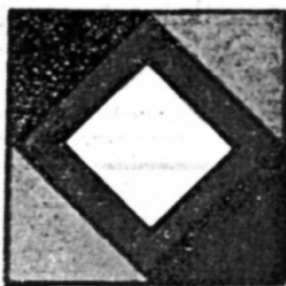

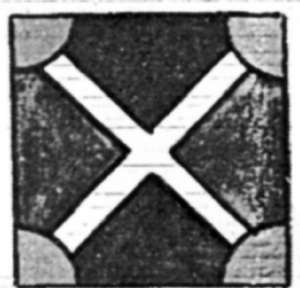

OTOÑO

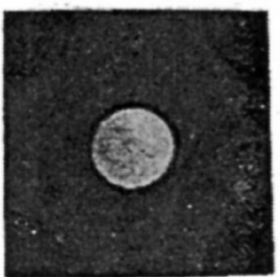

LA NOCHE

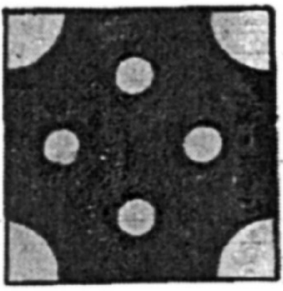

ESTRELLAS

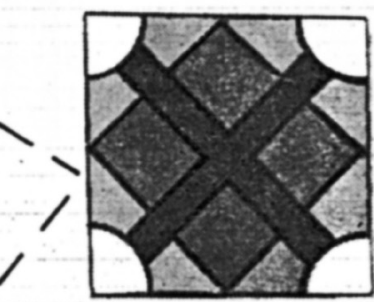

ECLIPSE

LUNA CRECIENTE 


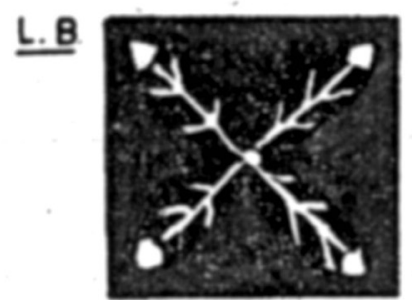

SAPAN INKA

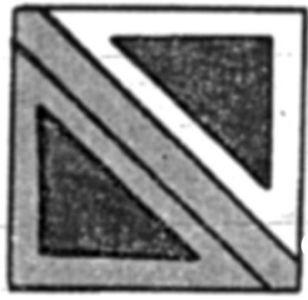

OCLLO

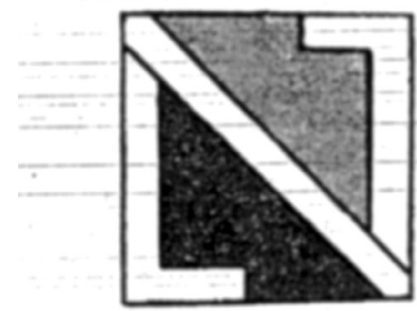

APU

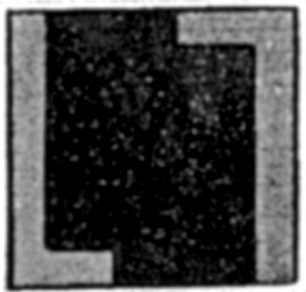

PALLA

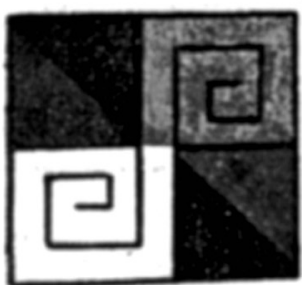

WILLKA?

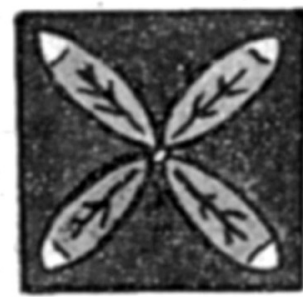

I NKA

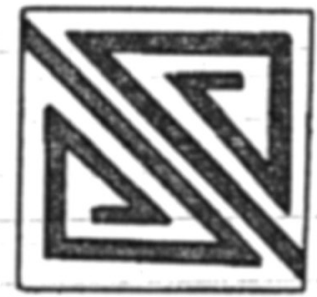

NUSTA

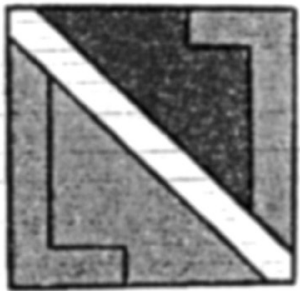

AUQUI?

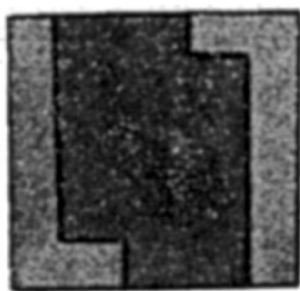

ACLLA
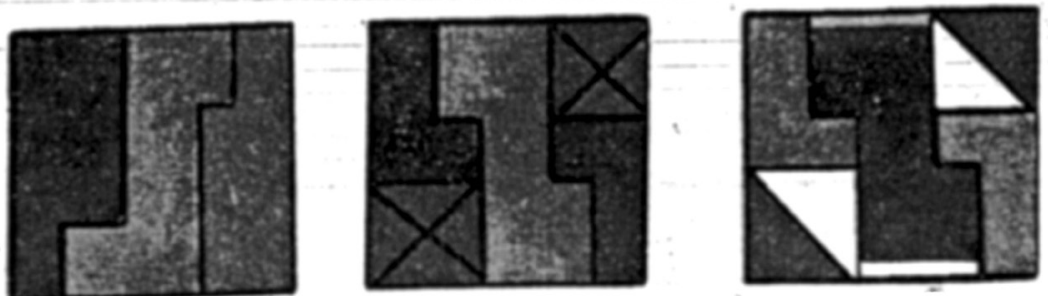

3 SIGNOS DE JERARQUIA RELIGIOSA 